\title{
Staggered Flux Phase in a Model of Strongly Correlated Electrons
}

\author{
J. B. Marston and J. O. Fjærestad \\ Department of Physics, Brown University, Providence, RI 02912-1843 \\ A. Sudbø \\ Department of Physics, Norwegian University of Science and Technology, N-7491 Trondheim, Norway
}

(Dated: February 11, 2002)

\begin{abstract}
We present numerical evidence for the existence of a staggered flux (SF) phase in the halffilled two-leg t-U-V-J ladder, with true long-range order in the counter-circulating currents. The density-matrix renormalization-group (DMRG) / finite-size scaling approach, generalized to describe complex-valued Hamiltonians and wavefunctions, is employed. The SF phase exhibits robust currents at intermediate values of the interaction strength.
\end{abstract}

PACS numbers: 71.10.Fd, 71.10.Hf, 71.30.+h, 74.20.Mn

The zero-temperature phase diagram of the twodimensional Hubbard model and its various extensions remains poorly understood. An intriguing possibility that has been the focus of considerable attention is known variously as the "orbital antiferromagnet" [1, 2, 3, the "staggered flux" (SF) phase 4, 5, 6, 7, 8, 9, or the "d-density wave" 10, 11]. The state breaks both time-reversal and lattice translation symmetries; another phase, the "circulating current phase" [12], is similar but preserves translational symmetry. Such "hidden" forms of order could arise in the pseudogap phase of the cuprate superconductors, implying the existence of a quantum critical point and possibly also non-Fermi liquid behavior 11, 12. The SF phase competes against other better-known phases such as charge-density waves (CDW), spin-density waves (SDW), superconductivity (BCS), stripes, and Fermi liquids. It is important to ascertain whether or not such order can really occur in sensible microscopic models of correlated electrons. In this Letter we study numerically the simplest possible system that could support such a phase, namely an extended version of the Hubbard model on a two-leg ladder (see Fig. 11). Our approach extends to arbitrary interaction strength earlier analytical work by two of us that showed that the SF phase arises at weak-coupling 13.

Ladder systems are of interest both because they are easier to understand theoretically than full twodimensional lattices, and also because they are realized in nature 14. Although ladders are one-dimensional, true long-range order (LRO) in the orbital currents is possible at zero temperature because the currents break discrete symmetries. This is in contrast to spin-density wave or BCS superconducting order which break continuous symmetries and which therefore can at most exhibit quasiLRO with power-law decay.

Away from half-filling, numerical studies of the t-J model 15, 16 and various extensions of it 17 on the twoleg ladder found no evidence for a SF phase. However, for weak interactions, tendencies towards SF ordering (not true LRO, but rather power-law decay of current-current
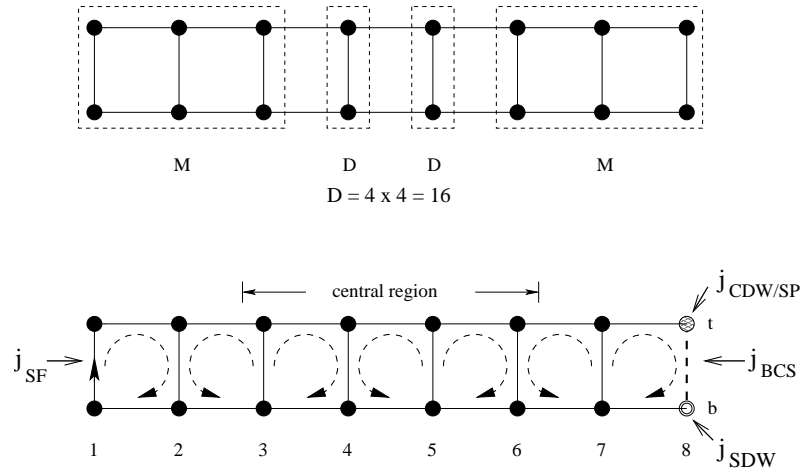

FIG. 1: Upper diagram: A two-leg ladder of length $L=8$. The left and right blocks each retain Hilbert spaces of dimension $M$. To implement the DMRG / finite-size algorithm, the two sites belonging to each rung are paired together into a single site with a Hilbert space of complex dimension $D=16$. Lower diagram: Source current $j_{S F}$ applied to the left-most rung induces currents in the interior of the ladder (dashed circles). A source of Cooper pairs $\left(j_{B C S}\right)$, a local chemical potential $\left(j_{C D W / S P}\right)$, and a local magnetic field $\left(j_{S D W}\right)$ are added at the ladder ends to induce BCS, SP, CDW, and SDW order in the central region of the ladder, which is then monitored as the ladder length increases.

correlation functions) have been found analytically both for the spinless [18] and spinful[19] cases (see also Ref. 20).

Here we will consider the half-filled two-leg ladder. We study an extended Hubbard model, the t-U-V-J model, defined on the two-leg ladder as follows: 


$$
\begin{aligned}
H & =\sum_{i=1}^{L-1} \sum_{\lambda=t, b}\left\{-t_{\|}\left(c_{i+1, \lambda}^{\dagger \sigma} c_{i, \lambda, \sigma}+H . c .\right)+V_{\|}\left(n_{i+1, \lambda}-1\right)\left(n_{i, \lambda}-1\right)+J_{\|} \vec{S}_{i+1, \lambda} \cdot \vec{S}_{i, \lambda}\right\} \\
& +\sum_{i=1}^{L}\left\{-t_{\perp}\left(c_{i, t}^{\dagger \sigma} c_{i, b, \sigma}+H . c .\right)+V_{\perp}\left(n_{i, t}-1\right)\left(n_{i, b}-1\right)+J_{\perp} \vec{S}_{i, t} \cdot \vec{S}_{i, b}\right\}+\frac{U}{2} \sum_{i=1, \lambda}^{L}\left(n_{i, \lambda}-1\right)^{2} .
\end{aligned}
$$

Here $c_{i \lambda}^{\dagger \sigma}$ creates an electron of spin $\sigma=\uparrow, \downarrow$ on site $i$ of $\operatorname{leg} \lambda=t, b$ of the ladder. Operators $n_{i, \lambda} \equiv c_{i, \lambda}^{\dagger \sigma} c_{i, \lambda, \sigma}$ and $\vec{S}_{i, \lambda} \equiv \frac{1}{2} c_{i, \lambda}^{\dagger \alpha} \vec{\sigma}_{\alpha}^{\beta} c_{i, \lambda, \beta}$ are respectively the number and spin at site $(i, \lambda)$. There is an implicit sum over repeated raised and lowered spin indices. Particle-hole symmetry implies that the chemical potential $\mu=0$ when the system is half-filled, with $\left\langle n_{i, \lambda}\right\rangle=1$ on each site.

For $V_{\|}=J_{\|}=0$ and $J_{\perp}=4\left(U+V_{\perp}\right)$ the model has $\mathrm{SO}(5)$ symmetry[21. The phase diagram of the $\mathrm{SO}(5)$-symmetric model with $t_{\perp}=t_{\|}$was investigated in Ref. 22 in the weak-coupling limit using a perturbative renormalization-group (RG) analysis combined with bosonization. One of the phases of the $\mathrm{SO}(5)$ model, located in the $U-V_{\perp}$ plane between the lines $V_{\perp}=-2 U$ and $V_{\perp} \approx-5.7 U$ with $V_{\perp}>0$ and $U<0$, was identified as having spin-Peierls (SP) order. However, a recent bosonization study by two of us 13 revealed that this phase in actuality exhibits no dimerization, but instead is the SF phase with true LRO in the orbital currents. For a correct understanding of the problem it was essential to treat carefully the "Klein factors" that must be introduced to maintain anti-commuting statistics of the (bosonized) fermionic degrees of freedom.

Previous RG studies have shown that in weak coupling a rather generic ladder model flows to a manifold with $\mathrm{SO}(5)$ symmetry for a range of values of the model parameters 22, 23. In agreement with these results, we have shown that there are RG flows towards the SF phase also when not too large non-SO(5)-invariant terms (such as $J_{\|}$and $\left.V_{\|}\right)$are added to the $\mathrm{SO}(5)$-model 24].

Weak-coupling RG is unreliable at intermediate interaction strengths. Instead we use the "infinitesize" version of the density-matrix renormalization-group
(DMRG) algorithm 25 to search for the ordered currents. The half-filled ladder is expected to be fully gapped over a large portion of the phase diagram; consequently (as shown below) the infinite-size algorithm is sufficiently accurate to obtain well-converged results. Each site of the ladder has a Hilbert space of dimension 4, as the site can either be unoccupied, have a single electron of either up or down spin, or be doubly occupied. To employ the DMRG algorithm, we group pairs of sites connected by a rung into a single site of Hilbert space dimension $D=4 \times 4=16$ (see Fig. 1). Errors in the calculation of observables introduced by the DMRG truncation of the Hilbert space can be systematically reduced by increasing the size $M$ of the Hilbert space retained in each of the two outer blocks up to limits imposed by computer memory and speed. The largest Hilbert space we use has $M=150$ with corresponding complex dimension $M \times D \times D \times M=5,760,000$.

Questions of the spontaneous formation of order are addressed by the combined DMRG / finite-size scaling approach described in some detail in Ref. 26. Quantumcritical points have been studied with the method, and critical exponents have been obtained at percent-level accuracy 26, 27]. In the present case we induce symmetry breaking by applying a source current $j_{S F}$ to the left end rung of the ladder. Terms that induce Cooperpair formation by breaking $\mathrm{U}(1)$ particle-number symmetry $\left(j_{B C S}\right)$, induce CDW and SP order by breaking lattice reflection symmetries $\left(j_{C D W / S P}\right)$, and induce a SDW through the application of a local magnetic field $\left(j_{S D W}\right)$, are also added to the right end of the ladder (see Fig. 1):

$$
\begin{aligned}
H \rightarrow H & +j_{S F} * i t_{\perp}\left(c_{1, t}^{\dagger \sigma} c_{1, b, \sigma}-H . c .\right)+j_{B C S} *\left(c_{L, t}^{\dagger \uparrow} c_{L, b}^{\dagger \downarrow}-c_{L, t}^{\dagger \downarrow} c_{L, b}^{\dagger \uparrow}+H . c .\right) \\
& +j_{C D W / S P} * n_{L, t}+j_{S D W} * S_{L, b}^{z} .
\end{aligned}
$$

In addition to these explicit source terms, we note that the open boundary conditions on the ladder also act as $\mathrm{O}(1)$ source terms for a columnar dimer pattern 26]. As the Hamiltonian is complex-valued when $j_{S F} \neq 0$, we generalize the DMRG code to handle complex-valued wavefunctions and reduced density matrices. This gener- 
alization comes at the cost of doubling the required memory, and slowing down both the sparse and the dense matrix diagonalization, but the cost is offset by the advantage that now we can access fully ordered ground states, well beyond linear response theory.

We calculate the expectation value of the current operators $2 t_{\perp} \operatorname{Im}\left\{c_{i, t}^{\dagger \alpha} c_{i, b, \alpha}\right\}$ and $2 t_{\|} \operatorname{Im}\left\{c_{i+1, \lambda}^{\dagger \alpha} c_{i, \lambda, \alpha}\right\}$ respectively on the central rungs and links of the ladder, checking that current is conserved (Kirchhoff's junction rule) at the vertices in the central region; deviations are typically of order $10^{-11}$ in units where $t_{\perp}=t_{\|}=1$. We also monitor the Cooper pair amplitude, $\left\langle c_{i, t}^{\dagger \uparrow} c_{i, b}^{\dagger \downarrow}-c_{i, t}^{\dagger \downarrow} c_{i, b}^{\dagger \uparrow}\right\rangle$; the average magnitude of deviations in the electron hopping amplitudes $\left\langle c_{i, \lambda}^{\dagger \sigma} c_{j, \lambda^{\prime}, \sigma}+H . c.\right\rangle$ from their mean value; deviations in the electron occupancy from one, $\left\langle n_{i, \lambda}-1\right\rangle$; and the local spin density, $\left\langle S_{i, \lambda}^{z}\right\rangle$.

First consider the pure minimal Hubbard t- $\mathrm{U}$ model with $t_{\perp}=t_{\|}=1, U=4$, and $V_{\perp}=V_{\|}=J_{\perp}=J_{\|}=0$. As shown in the semilog plot of Fig. 2(a), applied source currents of $j_{S F}=1$ and 0.01 induce currents on the four central rungs of the ladder that decrease exponentially as the ladder is enlarged via the DMRG algorithm, in agreement with the weak-coupling RG calculation 22. Likewise we find no instabilities towards BCS, SP, CDW or SDW order. Instead the phase is a fully-gapped insulator qualitatively the same as that found in the Heisenberg antiferromagnet.

Next consider the effect of turning on interactions $J_{\perp}$ and $V_{\perp}$ along the rungs of the ladder. According to the phase diagram in Ref. 22, for the case of exact $\mathrm{SO}(5)$ symmetry with $J_{\perp}=4\left(U+V_{\perp}\right)$ the SF phase should occur 13 in the weak-coupling limit for $U<0$ at $V_{\perp} / U=-9 / 4$. Fig. 2 shows that the $\mathrm{SF}$ phase does indeed arise at these ratios for intermediate interaction strength: For $U=-0.4, V_{\perp}=0.9$, and $J_{\perp}=2$, a small source current induces orbital currents in the central region of the ladder that grow with increasing ladder length. The currents saturate to the same non-zero asymptotic value in the limit of large ladder length, regardless of the size of the source terms. There is good convergence with increasing block Hilbert space dimension $M$; the asymptotic value of the rung current for the most accurate truncation of $M=150$ is 0.8047 and differs by only $0.1 \%$ from the value obtained with $M=100$. The currents alternate in sign from rung to rung as expected in the SF phase. There are no other instabilities: As shown in Fig. 3 the BCS, SP, CDW, and SDW order parameters all vanish exponentially as the ladder is enlarged via the DMRG algorithm, consistent with the weak-coupling prediction. The excitation spectrum remains fully gapped; consequently the SF phase exists over a range of parameter space well beyond the region of exact $\mathrm{SO}(5)$ symmetry. For example, robust currents arise for $U=-0.5, V_{\perp}=0.75$, and $J_{\perp}=2$, and also when interactions along the links are turned on,
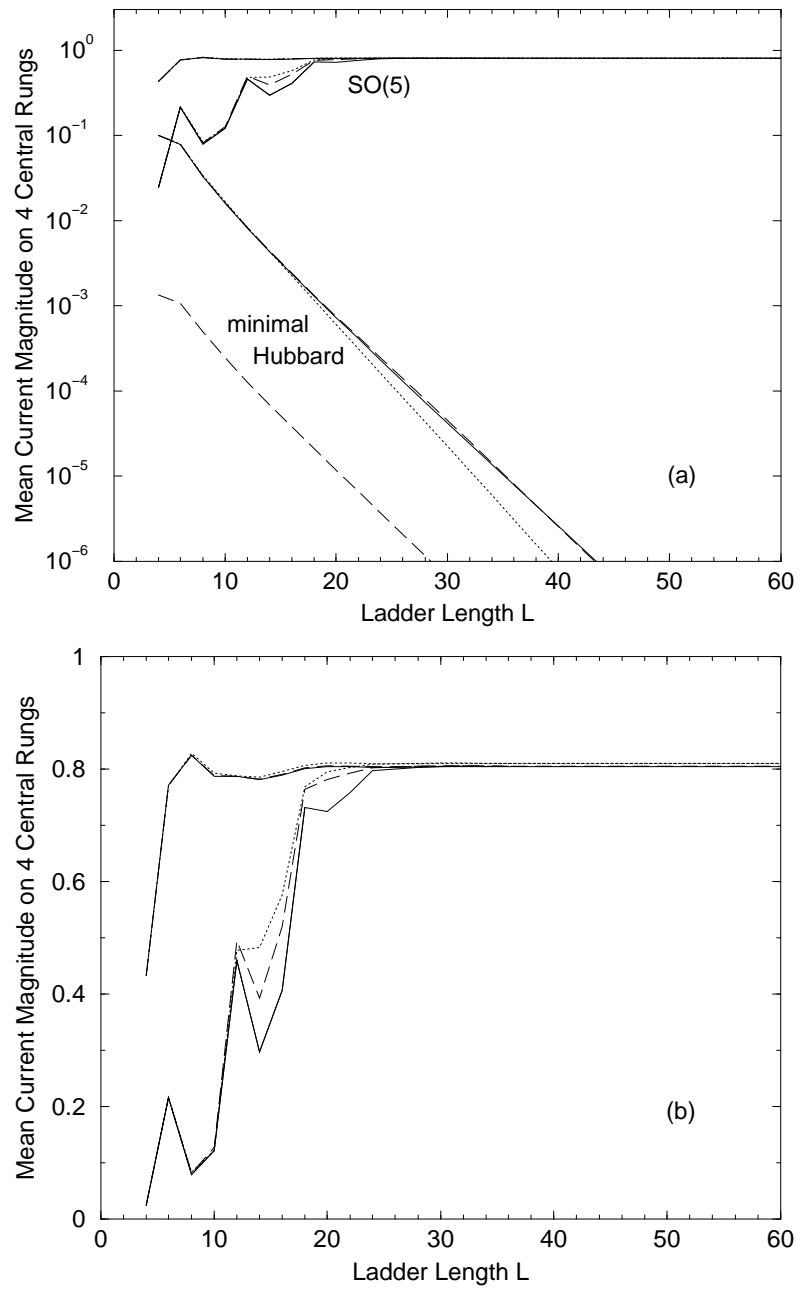

FIG. 2: Semilog (a) and linear (b) plots of the magnitude of the induced current averaged over the four central rungs of the ladder. Dotted lines are for calculations with $M=50$, dashed lines are for $M=100$, and solid lines are for $M=150$. Results are shown for the case of the $\mathrm{SO}(5)$ ladder with $t_{\perp}=t_{\|}=1$, $U=-0.4, V_{\perp}=0.9, J_{\perp}=2$, and $V_{\|}=J_{\|}=0$. Also shown on the semilog plot is the case of the minimal Hubbard model with $U=4$ and $j_{S F}=0.01$ (lower line that drops exponentially) and $j_{S F}=j_{B C S}=j_{C D W / S P}=j_{S D W}=1$ (upper lines that drop exponentially). In both plots, the lower set of $\mathrm{SO}(5)$ curves is for a small source current $j_{S F}=0.01$ applied to the ladder's left edge. The upper set is for large source currents $j_{S F}=j_{B C S}=j_{C D W / S P}=j_{S D W}=1$. Note the convergence to the same asymptotic value of current as the thermodynamic limit of long ladder length is approached. The asymptotic current for $M=100$ differs by $0.6 \%$ from that at $M=50$; the value for $M=150$ differs by only $0.1 \%$ from that at $M=100$ demonstrating good convergence.

$U=-0.5, V_{\perp}=V_{\|}=0.75, J_{\perp}=2$, and $J_{\|}=0.5$.

In the large- $J_{\perp}$ limit, with ratios $U / J_{\perp}=-1 / 4$ and $V_{\perp} / J_{\perp}=3 / 8 \mathrm{kept}$ fixed, the ground state of the ladder consists of a direct product of spin-singlet dimers on each of the rungs, with no broken symmetries 21. We have verified that the orbital currents cease when the interac- 


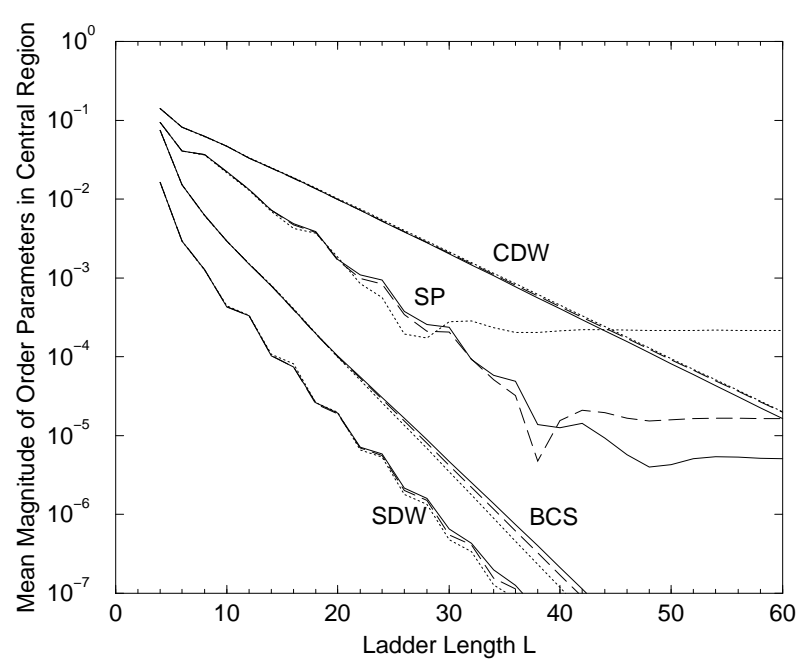

FIG. 3: Semilog plot of various order parameters in the SF phase (see text). Edge source terms $j_{B C S}=j_{C D W / S P}=$ $j_{S D W}=j_{S F}=1$ induce modulations in corresponding observables at the center of the ladder. Dotted, dashed, and solid lines correspond to $M=50,100$ and 150 respectively. Plotted in the figure, from highest to lowest in magnitude, are the order parameters for CDW, SP, BCS, and SDW tendencies. See text for the definition of these order parameters. Each of the expectation values decays exponentially with increasing ladder length. (The SP order parameter eventually levels off, but this residual order vanishes as the block size $M$ is increased, showing that it is an artifact of the Hilbert space truncation.) Similar results are obtained upon applying either large or small source terms, one at a time. Thus there is no tendency towards BCS, SP, CDW or SDW order in the SF phase.

tion strength is increased further to $J_{\perp}>2.5$. We are carrying out a more extensive investigation of the phase diagram of model Eq. 1 by using the weak-coupling RG equations in combination with the DMRG / finite-size scaling method 24.

It would be interesting to study the effects of doping away from half-filling. Numerical study of the doped SF phase would require the use of the more accurate "finitesize" DMRG algorithm, as either gapless excitations, or rich spatial structures such as stripes, are expected to occur 28. One question to be answered is whether or not LRO in the currents can still arise at commensurate hole concentrations.

In summary we have shown that a phase of strongly correlated electrons exists in which currents form spontaneously in the half-filled two-leg ladder. At half-filling the SF phase is fully gapped, and thus is an insulator of the Mott-Hubbard type. Our results, suitably generalized to two or three spatial dimensions, may have application to several stoichiometric compounds that were recently proposed to be in the SF phase 29. It is also intriguing that the SF phase lies in between a checkerboard CDW phase, and the D-Mott phase 13, 22, 24], as charge seg- ration into stripes and d-wave superconductivity are two phases that occur in the high- $\mathrm{T}_{c}$ cuprates.

Note added: U. Schollwöck has recently used the "finite-size" version of the DMRG algorithm, generalized to complex-valued wavefunctions, to study the $\mathrm{SO}(5)$ ladder with the same parameters as Fig. 2. The value of the saturated rung current that he finds agrees quantitatively with our result.

We thank I. Affleck, L. Balents, S. Chakravarty, S. Kivelson, D.-H. Lee, P. Lee, D. Scalapino, U. Schollwöck, M. Troyer, S.-W. Tsai, C. Varma, and S. White for helpful discussions. This work was supported in part by the NSF under grant Nos. DMR-9712391, CDA-9724347, and PHY99-07949. J.O.F. was supported by the Norwegian Research Council, Grant No 142915/432, and A.S. by Grant No. 115541/410 Some computational work was carried out at Brown University's Technology Center for Advanced Scientific Computing and Visualization.

[1] B. I. Halperin and T. M. Rice, Solid State Phys. 21, 115 (1968).

[2] H. J. Schulz, Phys. Rev. B39, 2940 (1989).

[3] A. A. Nersesyan and G. E. Vachnadze, J. Low Temp. Phys. 77, 293 (1989).

[4] I. Affleck and J. B. Marston, Phys. Rev. B37, 3774 (1988).

[5] J. B. Marston and I. Affleck, Phys. Rev. B39, 11538 (1989).

[6] T. C. Hsu, J. B. Marston, and I. Affleck, Phys. Rev. B43, 2866 (1991).

[7] D. A. Ivanov, P. A. Lee, and X.-G. Wen, Phys. Rev. Lett. 84, 3958 (2000).

[8] P. W. Leung, Phys. Rev. B62, R6112 (2000).

[9] K. Tsutsui, D. Poilblanc, and S. Capponi, Phys. Rev. B65, 020406 (2001).

[10] C. Nayak, Phys. Rev. B62, 4880 (2000).

[11] S. Chakravarty, R. B. Laughlin, D. K. Morr, and C. Nayak, Phys. Rev. B63, 094503 (2001).

[12] C. M. Varma, Phys. Rev. B55, 14554 (1997); Phys. Rev. Lett. 83, 3538 (1999); Phys. Rev. B61, 3804 (2000).

[13] J. O. Fjærestad and J. B. Marston, Phys. Rev. B65, 125106 (2002).

[14] E. Dagotto and T. M. Rice, Science 271, 618 (1996).

[15] D. J. Scalapino, S. R. White, and I. Affleck, Phys. Rev. B64 100506 (2001).

[16] J. B. Marston and A. Sudbø, unpublished.

[17] S. Chakravarty, U. Schollwöck, and M. Troyer, private communication (2001).

[18] A. A. Nersesyan, A. Luther, and F. Kusmartsev, Phys. Lett. A 176, 363 (1993).

[19] H. J. Schulz, Phys. Rev. B 53, R2959 (1996).

[20] E. Orignac and T. Giamarchi, Phys. Rev. B56, 7167 (1997).

[21] D. Scalapino, S.-C. Zhang, and W. Hanke, Phys. Rev. B58, 443 (1998).

[22] H. H. Lin, L. Balents, and M. P. A. Fisher, Phys. Rev B58, 1794 (1998).

[23] E. Arrigoni and W. Hanke, Phys. Rev. Lett. 82, 2115 
(1999).

[24] J. O. Fjærestad, J. B. Marston, and A. Sudbø, in preparation.

[25] S. R. White, Phys. Rev. Lett. 69, 2863 (1992); Phys. Rev. B48, 10345 (1993).

[26] S.-W. Tsai and J. B. Marston, Phys. Rev. B62, 5546 (2000).

[27] T. Senthil, J. B. Marston, and M. P. A. Fisher, Phys. Rev. B60, 4245 (1999).
[28] S. R. White, I. Affleck, and D. J. Scalapino, Phys. Rev. B65, 165122 (2002).

[29] P. Chandra, P. Coleman, and J. A. Mydosh, Physica B 312-313, 397 (2002); P. Chandra, P. Coleman, J. A. Mydosh, and V. Tripathi, cond-mat/0205003 (to appear in Nature); D. F. Schroeter and S. Doniach, condmat/0201524. 